\title{
Levels of interleukin 4 and immunoglobulin $E$ in umbilical cord blood from children born to allergic mothers
}

\begin{abstract}
Objective: The objective of this research is to determine the levels of Interleukin 4 and Immunoglobulin E in umbilical cord blood of healthy subjects born to mothers with a history of allergy, born in the Maternity of Dr. Carlos Arvelo Military Hospital and its linkage with the appearance of allergic diseases in the first trimester of life.

Methods: A descriptive, exploratory and non-experimental study was carried out considering all the children of allergic mothers. Five (5) $\mathrm{ml}$ of blood was drawn from the subject's umbilical cord with a sterile injector; then three (3) $\mathrm{ml}$ of serum was collected by centrifugation. Three (3) $\mathrm{ml}$ of blood was also extracted from the mother to process the levels of Immunoglobulin E. Measurements of Interleukin $4(\mathrm{Pg} / \mathrm{ml})$ and Immunoglobulin E (IU) was performed by the Indirect Elisa Technique. To evaluate levels of Interleukin 4 , detectable and non-detectable categories were used; the referential value was $0,2 \mathrm{Pg} /$ $\mathrm{ml}$. Values lower than 0,2 were considered no detectable and values higher than $0,2 \mathrm{Pg} /$ $\mathrm{ml}$ were considered detectable. A 50-200 IU referential value was considered to evaluate Immunoglobulin E levels in mothers; as for the newborns values higher than $10 \mathrm{IU}$ and lower than 10 UT were used as referential values for Immunoglobulin E. The patients were followed-up of in the first three months of postnatal life.

Results: 62 subjects were studied, 42 remained in the experimental group. 2 subjects had total IgE levels in umbilical cord blood with levels higher than $10 \mathrm{IU}$, only one of them belonged to the experimental group and the other was from the control group. More than a half of the 42 subjects in the experimental group had detectable levels of IL4. 16 subjects finally completed the study; 12 belonged to the experimental group, more than half had detectable levels of IL4 in the umbilical cord blood at the third month. All subjects had total $\mathrm{IgE}$ levels in the umbilical cord blood higher than 10IU, half of the subjects had equally elevated levels in the third month, with no significant correlation with maternal IgE values. 6 subjects from the experimental group had allergic manifestations of atopic dermatitis and so did 2 from the control group.

Conclusion: In the experimental group, mothers had high levels of $\operatorname{IgE}$, but there is not any association with high levels of IgE in the umbilical cord blood of their children, which could be explained by the high specificity but low sensitivity of IgE. Subjects with detectable levels of interleukin 4 in umbilical cord blood persisted with detectable values at three months of life, suggesting that these values are much more sensitive as predictors of future allergy development.
\end{abstract}

Keywords: immunoglobulin E, interleukin 4, allergic diseases, atopy
Volume 5 Issue 6 - 2017

\section{Rodr guez Alvarez LigiaAurora, Zabala Galet Mayerling Mayerling ,Tocuyo Janine DelValle, Pellizzeri Sanchez AuraEstela \\ Medical Specialists Allergology and Immunology, Service Military Hospital,Venezuela}

Correspondence: Rodríguez Alvarez Ligia Aurora, Immunology Institute, UCV, Universidad Central de Venezuela, Facultad de Medicina, Hospital Universitario de Caracas, Urbanización Los Chaguaramos, Caracas, Venezuela,, Tel 580-412-227-31 I-5, Email auroradoct@gmail.com; auroradoct@yahoo.es

Received: January 01, 1970 | Published: July 14, 2017

\section{Introduction}

The prevalence of allergic diseases, such as asthma, rhinoconjunctivitis and atopic dermatitis, have dramatically increased in industrialized countries in recent years; this has a significant impact on public health. ${ }^{1,2}$ Because allergic diseases are generally diagnosed before six years old, ${ }^{3}$ early detection of atopy should lead to develop prevention means and treatment of these conditions in those individuals at risk. Elevated levels of Immunoglobulin E in umbilical cord blood have been reported to be directly related to the development of atopy in children, particularly those with family records of allergic diseases. ${ }^{4,5}$ Also, recent studies indicate that exposure to innocuous allergens early in life, even before birth, may influence the incidence of allergic diseases later in life. ${ }^{6,7}$ During pregnancy, the maternal-fetal interface is surrounded by high levels of Th2-pattern cytokines, possibly favoring the development of the Th2 immune response -as in children born to these mothers; thus maternal immunity status may affect the profile Th1/Th2 in the newborn. ${ }^{6}$ The development of allergic disease is associated with a more marked deviation towards the Th2 profile at birth, such as increased levels of Interleukin 4 and Immunoglobulin E. ${ }^{6}$ The present article aims to determine the levels of these molecules in neonates born to mothers with records of allergic diseases and their probable predictive value of allergic disease development in infants. ${ }^{7}$

\section{Theoretical framework}

The development of allergic pathology occurs as a consequence of an alteration of natural immunity, and as an alteration of the corporal protection mechanisms. This rises an erroneous or exaggerated response against normally innocuous antigens which generates clinical frames with skin, digestive and (or) respiratory manifestations; those manifestations are responses mediated by Immunoglobulin E.8 Since the 1970 s, there has been a marked increase in the frequency of this pathology in population; therefore, a greater interest in the study of the mechanisms involved has been observed..$^{8}$ In the late $1960 \mathrm{~s}$, Immunoglobulin E ( $\mathrm{IgE}$ ) was discovered as a new class of antibodies capable of transferring sensitivity to allergens. Immunoglobulin 
E antibodies are involved in allergic inflammatory processes such as anaphylaxis, allergic rhinitis, asthma and atopic dermatitis or eczema. Immunoglobulin E antibodies have also been associated with protection against parasitic infections. Studies in Gambia revealed that high levels of parasite-specific IgE were associated with this infection resistance. Immunoglobulin $\mathrm{E}$ is the least frequent antibody present in serum, with a normal concentration of 50-200 IU in non-allergic individuals. Serum Immunoglobulin E half-life was 3 days; however, much of Immunoglobulin E remains in the tissues. In contrast, Serum Immunoglobulin E bound to the cell receptor has half-life of a few weeks.

The Immunoglobulin E expression is produced out of a heavy chain change from an Immunoglobulin M; this is caused by a somatic recombination of the genes in the germ line of B cells, in a micro Th2 environment represented by Interleukin 4, 5, 9 and 13. It is structurally similar to other classes of antibodies, with two identical heavy chains and two light chains with constant and variable regions consisting of immunoglobulin domains. In contrast to Immunoglobulin $\mathrm{G}$ antibodies, which have 3 constant domains in the heavy chain, Immunoglobulin $\mathrm{E}$ has 4 domains without hinge region which makes it less flexible. It also has a higher content of glycosylation sites than Immunoglobulin G. ${ }^{9}$ Three IgE receptors have been identified in humans up to date. Within the human body, most IgE binds to its high affinity receptor (FCER1) which is mostly present in mast cell and basophils, but it is also found on antigen-presenting cells (CPAs) such as Langerhans cells. FCeR1 are constituted by 4 chains in mast cells and basophils and by 3 chains in monocytes, macrophages and neutrophils. Cross-linking of FC\&R1 through IgE activates mast cells and basophils releasing proinflammatory mediators such as histamine, leukotrienes, and cytokines. Positive regulation of additional FCER1 is also triggered. The low affinity IgE receptor (FCERII) (CD23) is expressed on some cell types including $\mathrm{B}$ cells activated $\mathrm{T}$ cells, monocytes, eosinophils, platelets, follicular dendritic cells and some thymic epithelial cells. After IgE binding to the antigen, CD23 facilitates the antigen presentation to T cells; this also works as a negative feedback on the regulation of IgE production. There is also a third IgE receptor originally called $\varepsilon$-binding protein, now known as galactin-3. This receptor presence has been reported on neutrophils; there, it plays a role in cellular activation and it is also present on trophoblast cells in the placenta.

In mice, there is a fourth IgE receptor called FccRIV which has been studied because of its high homology to the FccRIIIA human receptor; it was first suggested as an equivalent to the human receptor. Recent studies, however suggest that the human equivalent of this receptor is FceRI. Immunoglobulin E mediation is regulated by Th1 lymphocyte response represented by interleukin 2 and gamma interferon, which inhibit its production; it is also regulated by Th-2 lymphocyte response formed by interleukin-4, interleukin-10 and interleukin-13 which stimulate production of Immunoglobulin E. ${ }^{8}$ The placenta is a fetomaternal organ; one of its main functions is to facilitate the transfer of nutrients, oxygen and immunological factors from the mother and the fetus and waste products from the fetus to the mother. It is a highly vascularized tissue, where fetal blood flows through a network of umbilical capillary vessels located within the branched chorionic, terminal vessels; maternal blood flows through the intervillous space without involving the capillary network. The development of the placenta begins with the implantation of the blastocyst in the endometrium, which is called decidua during pregnancy. The cells of the extracellular trophoblast invade the fetal blood vessels in lacunar decidua, and then what will later shape the intervillous space begins to develop. The decidua contains approximately $40 \%$ of the immune cells and this is crucial for the development of the placenta. Most of the leukocyte subpopulations are uterine natural killer (UNK) cells, macrophages, dendritic cells, T cells and NKT cells. Both T cells, CD4 + and CD8 + cells are present in the decidua. Some CD $4+$ cells are Foxp 3 and they express regulatory $\mathrm{T}$ cells and those believed to be important to uphold maternal-fetal tolerance. Induction of tolerance by regulatory $\mathrm{T}$ cells can be generated throughout decidua dendritic cells. One mechanism that has been proposed is the induction of dendritic cells to express indolamine 2, 3-dioxygenasa. ${ }^{9}$ External cells of the trophoblast covering the villi (syncytiotrophoblast) are the main site of nutrients and gas exchange between the maternal blood and the fetus. Together with the endothelium of the fetus blood vessels they compose the placental barrier. This layer of trophoblast cells is a syncytium and can only be passed through transcellularity. From the villi and in the fetus, the transport is performed by paracellularity or transcellularity; this layer crosses the endothelium of the fetal blood vessels. Apart from fetal vessels and trophoblast cells, the villi also contain mesenchymal cells and fetal macrophages called Hofbauer cells, as well as small amounts of fibroblasts, myofibroblasts and mast cells. In contrast to the decidua, villi usually contain very few lymphocytes.

Concerning the ability of an altered prenatal Immunoglobulin E response in children predisposed to allergic diseases, elevated levels of Immunoglobulin E in cord blood have proven to present high specificity but very low sensitivity. However, several studies have shown that the pattern of allergen response of newborn mononuclear cells allows predicting the subsequent development of allergic diseases. It is important to note that this response has been found from the twenty-second week of gestation. It has also been shown that the specific reactivity to birch pollen at birth is more likely if exposure to this allergen occurred between the twentieth and twenty-eighth week of gestation, a period in which the fetus would be more sensitive to specific allergen reactivity. ${ }^{12}$

Pregnancy is associated with the development of various physiological, hormonal and immunological changes. Studies on serum immunoglobulin levels are limited in number and have shown inconsistent results. IgG levels decrease significantly. The fall in serum IgG levels can be attributed to hemodilution during pregnancy and to the transplacental transfer of maternal IgG to the fetus. Serum IgA has been shown to have no change. Similarly, IgM in serum has been shown to have no change, total serum IgE level is used as a general marker of atopy, although it is neither inclusive nor exclusive. However, the basal level of total serum IgE is influenced by many factors such as genetics, age, race, season, type of infant feeding and smoking. Studies on the effect of pregnancy on total serum IgE are limited. ${ }^{9,10}$ Serum IgE has been shown to have a major change. Pregnant asthma patients who had an increase or changes in IgE were found to have a tendency to exacerbate asthma. Thus, it is observed an increase in $\operatorname{IgE}$ levels in the postpartum month in comparison with the third trimester results. During the first months of life, a high percentage of atopic individuals may manifest allergic disease; in general, atopic dermatitis is the primary manifestation. Children with onset atopic dermatitis in lactation -in addition to a family record of allergy have a higher risk of subsequent development of asthma and (or) other allergic manifestations. ${ }^{12}$

The hygiene hypothesis tries to explain the increase in the prevalence of atopic diseases in recent years. Neonates have an immunological response with a Th2 phenotype and early infections would be crucial to tilt the response to the Th1 pattern. According to the hygiene hypothesis, less exposure to germs, in association with 
a new lifestyle, would explain the increase in allergic diseases; the hypothesis is strongly supported by the fact that residence in rural settings in the early years reduces the risk of atopy, asthma and rhinitis. Early exposure to bacterial pathogens decreases the risk of allergy. In relation to viruses, the effect is not so homogeneous, some are associated with protection, while others (rhinovirus and respiratory syncytial virus) initiate or aggravate asthma, in turn the decrease of lactobacilli and bifidobacteria in intestinal levels (due to antibiotic use) increases the risk of atopy. There are other maternal factors and some other factors associated with the first years of life that influence an environment, promoting allergic diseases such as vitamin D. Vitamin D has effects on the immune system acting on B and $\mathrm{T}$ lymphocytes, monocytes, macrophages and dendritic cells -that have receptors for this vitamin which modulate the levels of several cytokines. Deficiency of this vitamin is associated with increased risk of allergic and autoimmune diseases. In addition, inadequate maternal nutrition during gestation seems to be associated with unfavorable effects on the production of allergies, while zinc and antioxidants would be protective. Children who develop eczema have significant disorders in epidermal barrier function as a consequence of mutations in filaggrin, a key protein for aggregation and keratin cross linking; as a result, there is greater absorption of allergens and less resistance to infections and to local and systemic inflammation with a Th2 pattern. ${ }^{10}$

On the other hand, only $20 \%-40 \%$ of those with allergic rhinitis will develop asthma in the future. Several immunological markers have been studied as predictors for the development of atopy, they are: levels of total Immunoglobulin E in umbilical cord blood; levels of serum Immunoglobulin E in infancy; serum eosinophilia in childhood; eosinophilic cationic protein and levels of Interleukin 4; serum determination of interferon-gamma. In the early stages of gestation (11 th week), it is possible to detect the production of Immunoglobulin E in the fetus liver and lungs; the clinical relevance of these findings is unknown. ${ }^{7}$ Most allergy and care researchers agreed with the need of an early diagnosis of allergic diseases since early treatment and good prognosis of allergic diseases especially respiratory diseases depend on that. ${ }^{6,13}$ The determination of Immunoglobulin $\mathrm{E}$ in cord blood is a valuable parameter directly related to the newborn. ${ }^{6}$ The rate of cord Immunoglobulin $\mathrm{E}$ and the family record of atopy have been correlated with the occurrence or non-occurrence of allergic diseases. The number of children controlled and followed up to 18 months in the shortest periods and up to 8 years in the long ones is very high. Studies in Europe and the United States report similar conclusions, ${ }^{10}$ which give a high value to the following conclusions: up to $30 \%$ of children with positive atopic family records and high Immunoglobulin E in umbilical cord blood will develop Atopy. If the family record is positive and Immunoglobulin $\mathrm{E}$ in umbilical cord blood is normal, the development of atopy also occurs in $30 \%$. Only $10 \%$ of children with a negative family record and a raised Immunoglobulin E in umbilical cord blood will present atopy. It is, therefore, conclusive, that the family record (the inherited genetic component) is the most reliable predictor and that Immunoglobulin E in umbilical cord by itself is a parameter of very low sensitivity as a predictive factor. Recent studies have shown that maternal asthma records correlates with an increase in Immunoglobulin E in umbilical cord blood and this was associated with a fourfold higher risk of food allergy development in the first year of life. ${ }^{4}$

Other markers in umbilical cord blood that have been studied to evaluate predictors of allergy are: eosinophil count study; suppressor T lymphocyte decline; TH2 lymphocyte augmentation; determined phenotypes $\alpha$-1-antitrypsin, Immunoglobulin $\mathrm{G}$ anti- $\beta$-lactoglobulin specific antibodies; in relation to the regulation of Immunoglobulin
E production, studies are interested on Interleukin 4 and Interferon gamma in umbilical cord blood., ${ }^{5,14}$ More recent investigations also study interleukin-2, T4 and T8 lymphocyte populations and Interferongamma correlated with Immunoglobulin E in umbilical cord rate with no conclusive results. ${ }^{15}$ Other atopy predictors have been used, such as lymphocytes population's studies, Immunoglobulin E levels during the first year of life, serum eosinophilia and some clinical aspects evaluation such as the recurrent laryngitis occurrence with a 58\% risk, unidentified cause wheezing (36\%). In addition to wheezing, when eosinophilia is detected in blood the risk of allergy is $75 \% .{ }^{16}$ Among the genetic aspects listed as predictors of allergic disease, studies have considered male sex, atopy family records, chromosomes 5, 16, 6 and 11 markers. ${ }^{14}$

\section{Problem statement}

The accelerated rise in allergic diseases worldwide ${ }^{2}$ and the evidence that these diseases are generally manifested in infants and preschoolers has generated an interest in the scientific community to detect early patients at developing atopic disease risk; this leads to implement measures of prevention and treatment of these conditions in those individuals at risk. There is no single parameter that makes possible to accurately diagnose allergic diseases in the neonate, gestation or lactation period, so a combination of anamnesis, clinical records and exploration and laboratory tests could allow making an early diagnosis. ${ }^{17}$ Because the fetus is able to produce its own Immunoglobulin $\mathrm{E}$ from the second trimester, and intrauterine environmental factors influence the deviation of the cytokine pattern towards a TH2 response, ${ }^{6}$ we propose to quantify the levels of Immunoglobulin E and Interleukin 4 in umbilical cord blood to evidence its predictive value of allergic disease.

\section{Problem delimitation}

Two previous Venezuelan studies ${ }^{16}$ have investigated the predictive value of Immunoglobulin E levels in umbilical cord blood. However, levels of Interleukin 4 as the main modulating cytokine of the Th2 response pattern have not been evaluated in previous studies. The present investigation aims to determine the predictive value of objective parameters such as a family record and laboratory immuno serological-such as Immunoglobulin E and Interleukin 4 in determining the risk of a newborn to develop allergic pathology. The relevance of this study remains in its predictive value determination, from pregnancy, out of umbilical cord blood samples from children born to allergic mothers. The aim is to develop an early allergic processes diagnosis.

\section{General objective}

Determine the levels of Interleukin 4 and Immunoglobulin $\mathrm{E}$ in umbilical cord blood of healthy subjects born to mothers with allergic records- who were born in the Military Hospital Maternity, Dr. Carlos Arvelo, between March 1, 2011 and May 31, 2011 in order to link first trimester of life to allergic disease's appearance.

\section{Specific objectives}

Quantify levels of Interleukin 4 and Immunoglobulin E in umbilical cord blood in neonates born to mothers with allergic diseases records. Determine the influence of maternal antecedents in Interleukin 4 and Immunoglobulin E levels in newborn umbilical cord blood. Monitor once a month -in postnatal control allergic disease occurrence during the first three (3) months of life. Quantify Interleukin 4 and Immunoglobulin E levels in the subjects at three (3) months of life. Establish the relationship between the values of Interleukin 4 and 
Immunoglobulin $\mathrm{E}$ in the development of allergic diseases during the first three (3) months of life.

\section{Materials and methods}

\section{Type of study}

This research aims to determine the levels of Interleukin 4 and Immunoglobulin $\mathrm{E}$ in umbilical cord blood of children born to allergic mothers; the methodology involves descriptive and exploratory processes ${ }^{18}$ aimed to collect information related to the levels of Interleukin 4 and Immunoglobulin E in children collected umbilical cord blood at the time of birth. The research is considered non-experimental because it doesn't look forward to establish the phenomenon causes, ${ }^{18}$ but to describe the variable (Interleukin 4 and Immunoglobulin E) which is not manipulated; thus the data are collected in a same time and in the same space.

\section{Methodology}

Once the subjects were selected, specific data were collected. Perinatal data from the medical history included: personal data gestation and childbirth information; allergic family background information about allergic diseases in first and second line relatives that allows associating the genetic load influence on allergic disease development; information about non-allergic maternal pathologies during pregnancy which may give false positives to the study; data related to allergic maternal pathologies before pregnancy, which confirm the contribution of genetic load to the subject allergic diseases; mother allergic pathologies during pregnancy, which could suggest an increased risk of allergy to the subject; and allergenic food consumption during pregnancy that can influence the development of allergies in the subject during the first two years of life. So, specifically collected data related to that information contained: pregnancy complications data to gather the possible maternal diseases during gestation that could affect the subject; perinatal anthropometric data on the subject at birth (including values of Interleukin 4 and Immunoglobulin $\mathrm{E}$ in umbilical cord blood and maternal Immunoglobulin E measurement); emergence of allergic disease during the first three postnatal month's data which included allergic diseases developed during the postnatal period. That information was correlated with the values of Interleukin 4 and Immunoglobulin $\mathrm{E}$ at birth.

Additionally, pathology-allergen relationship data (involving allergic disease manifestations occurrence) was related to food allergens during the period of observation. A monthly check during the first trimester of life was performed. Infant feeding information containing the subject ingested food in the first three months of the postpartum period and the development of adverse reactions to it was then collected. Mother's diet gathered data included food ingested by the mother during the first three postnatal months when food allergenicity development through breastfeeding was studied. An indirect type Elisa technique for postnatal measurement of Interleukin 4 and Immunoglobulin E values on the subject was applied in the first three months of postnatal life and this was correlated with the values at birth. Five (5) $\mathrm{ml}$ of blood was drawn from the subject's umbilical cord with a sterile injector; the sample was placed in a tube without anticoagulant, it was kept in the rack for 30 minutes; then three (3) $\mathrm{ml}$ of serum was collected by centrifugation. After the necessary time the serum was drawn with a pipette and divided into two equal aliquots which were placed in two (2) test tubes labeled with the subject's data and frozen at $-20{ }^{\circ} \mathrm{C}$ until it was processed. Three (3) $\mathrm{ml}$ of blood was also extracted from the mother to process the levels of Immunoglobulin E. Measurements of Interleukin 4 (Pg/ $\mathrm{ml}$ ) and Immunoglobulin E (IU) was performed by the Indirect Elisa
Technique. To evaluate levels of Interleukin 4 , detectable and nondetectable categories were used; the referential value was $0,2 \mathrm{Pg} / \mathrm{ml}$ (3). So values lower than 0,2 were considered non detectable and values higher than $0,2 \mathrm{Pg} / \mathrm{ml}$ were considered detectable. A 50-200 IU referential value was considered to evaluate Immunoglobulin $\mathrm{E}$ levels in mothers; as for the newborns values higher than $10 \mathrm{IU}$ and lower than 10 UT were used as referential values for Immunoglobulin E.

\section{Evaluation and monitoring criteria}

The inclusion criteria for subjects were:

a. Motherhood: Mothers with allergic diseases record (asthma, rhinitis, rhino-conjunctivitis or atopic dermatitis) were selected as well as those controlled during pregnancy.

b. Newly born: healthy newborns and newborns at term (according to gestational age) subjects without knowing intrauterine pathologies were chosen.

The exclusion criteria for subjects were

a. Newly born: newborn subjects with known intrauterine or neonatal pathologies (Intrauterine Growth Retardation) and newborn subjects with low birth weight were omitted.

b. Motherhood: mothers with pathologies other than allergic diseases were excluded.

\section{Statistical methods}

Basic descriptive statistics were applied to process and analyze data in order to characterize the group according to quantitative variable. Likewise, the percentages were calculated from occurrence frequency according to categorical variables. Variable association tests $(\chi 2)$ were applied to observe possible relationships. In all cases a value of $\mathrm{p} \leq 0.05$ was considered significant. SPSS version 19 Statistical Package was used to process data (Tables 1-12).

Table I Experimental group percentage distribution according to type of maternal background

\begin{tabular}{lll}
\hline Type of maternal background & Experimental & \\
\hline Asthma & $\mathbf{N}$ & $\%$ \\
Rhinitis & 10 & 27,03 \\
Asthma and rhinitis & 14 & 37,84 \\
Atopic dermatitis & 8 & 21,62 \\
Rhinitis and atopic dermatitis & $\mathrm{I}$ & 2,70 \\
Asthma and urticarial & 2 & 5,41 \\
Rhinitis and urticarial & $\mathrm{I}$ & 2,70 \\
TOTAL & $\mathrm{I}$ & 2,70 \\
\hline
\end{tabular}

From experimental group, maternal allergic background, almost half indicated bronchial asthma and rhinitis pathology.

\section{Analysis and interpretation of results}

12 of the 16 subjects who completed the study belonged to the experimental group; more than half had detectable IL4 levels in umbilical cord blood and at the third month control. All had total IgE levels in cord blood higher than $10 \mathrm{IU}$; half of the subjects presented equally elevated levels in third month control but there is no significant correlation with maternal IgE values. 6 of the 12 subjects who were in the experimental group had higher detectable IL4 levels in umbilical cord blood and in control sample at the third month of life. Total IgE levels in umbilical cord blood were higher than $10 \mathrm{IU}$, but there was no significant correlation with maternal $\operatorname{IgE}$ values. 6 subjects from the experimental group and 2 subjects of the control group had allergic manifestations such as atopic dermatitis. 
Table 2 Subject group percentage distribution according to type of complications during pregnancy

\begin{tabular}{|c|c|c|c|c|c|c|}
\hline \multirow{2}{*}{ Complications in pregnancy } & \multicolumn{2}{|c|}{ Experimental } & \multicolumn{2}{|c|}{ Control } & \multicolumn{2}{|c|}{ TOTAL } \\
\hline & $\mathbf{N}$ & $\%$ & $\mathbf{N}$ & $\%$ & $\mathbf{N}$ & $\%$ \\
\hline ITU & 7 & 70,00 & 3 & 30,00 & 10 & 28,57 \\
\hline Retroplacental hematoma & 1 & 100,00 & 0 & 0,00 & I & 2,86 \\
\hline Urinary infection & 13 & 86,67 & 2 & 13,33 & 15 & 42,86 \\
\hline Vaginal infection & I & 20,00 & 4 & 80,00 & 5 & 14,29 \\
\hline Threatened Abortion & I & 100,00 & 0 & 0,00 & I & 2,86 \\
\hline Asthma and urticaria & 2 & 100,00 & 0 & 0,00 & 2 & 5,71 \\
\hline Leucorrhea & I & 100,00 & 0 & 0,00 & I & 2,86 \\
\hline TOTAL & 26 & 74,29 & 9 & $25,7 \mid$ & 35 & 100,00 \\
\hline
\end{tabular}

Experimental group most frequent complication was an infection of the urinary tract; but 2 subjects had allergic conditions such as bronchial asthma and urticaria.

Table 3 Subject group percentage distribution according to type of complications during childbirth

\begin{tabular}{lllllll}
\hline \multirow{2}{*}{ Complications in labor } & \multicolumn{2}{l}{ Experimental } & \multicolumn{2}{c}{ Control } & \multicolumn{2}{c}{ TOTAL } \\
& $\mathbf{N}$ & $\%$ & $\mathbf{N}$ & $\%$ & $\mathbf{n}$ & $\%$ \\
\hline Asthma & 2 & 100,00 & 0 & 0,00 & 2 & 33,33 \\
Leucorrhea & $\mathrm{I}$ & 100,00 & 0 & 0,00 & $\mathrm{I}$ & 16,67 \\
Obstetric Trauma & $\mathrm{I}$ & 100,00 & 0 & 0,00 & $\mathrm{I}$ & 16,67 \\
DFP Caesarean section & $\mathrm{I}$ & 100,00 & 0 & 0,00 & $\mathrm{I}$ & 16,67 \\
Breech presentation & 0 & 0,00 & $\mathrm{I}$ & 100,00 & 1 & 16,67 \\
TOTAL & 5 & 83,33 & $\mathrm{I}$ & 16,67 & 6 & 100,00 \\
\hline
\end{tabular}

Only 5 patients from experimental group had complications during childbirth and 2 of them had asthma.

Table 4 Subject Group Percentage Distribution According to Presence of Maternal Allergic Records and lgE Levels in Umbilical Cord

\begin{tabular}{|c|c|c|c|c|c|c|}
\hline \multirow{2}{*}{ Maternal allergic record } & \multicolumn{2}{|c|}{ IgE cord(Low Values - I0) } & \multicolumn{2}{|c|}{ IgE cord (High Values +10$)$} & \multicolumn{2}{|c|}{ Total } \\
\hline & $\mathbf{N}$ & $\%$ & $\mathbf{N}$ & $\%$ & $\mathbf{N}$ & \\
\hline$\overline{\mathrm{Si}}$ & 36 & 97,30 & I & 2,70 & 37 & 59,68 \\
\hline No & 24 & 96,00 & I & 4,00 & 25 & 40,32 \\
\hline TOTAL & 60 & 96,77 & 2 & 3,23 & 62 & 100,00 \\
\hline
\end{tabular}

In this case, the 2 subjects with IgE levels higher than $\mathrm{I} 0 \mathrm{IU}$ in umbilical cord blood gad no maternal allergic record.

Table 5 Subject group percentage distribution according to IgE levels in umbilical cord and maternal IgE levels

\begin{tabular}{|c|c|c|c|c|c|c|}
\hline \multirow{2}{*}{ IgE Cord (Categories) } & \multicolumn{2}{|c|}{ Maternal IgE (Low Values - I 50) } & \multicolumn{2}{|c|}{ Maternal IgE (High values + I50) } & \multicolumn{2}{|c|}{ Total } \\
\hline & $\mathbf{N}$ & $\%$ & $\mathbf{N}$ & $\%$ & $\mathbf{N}$ & $\%$ \\
\hline Valores bajos $(-10)$ & 57 & 95,00 & 3 & 5,00 & 60 & 96,77 \\
\hline Valores elevados $(+10)$ & 0 & 0,00 & 2 & 100,00 & 2 & 3,23 \\
\hline TOTAL & 57 & 91,94 & 5 & 8,06 & 62 & 100,00 \\
\hline
\end{tabular}

Only 2 of the 5 mothers with Total IgE levels above $150 \mathrm{IU}$ were directly related to their Total IgE levels in cord blood. More than half of the 42 subjects in the experimental group had detectable levels of IL4.

Table 6 Subject group percentage distribution according to IL4 levels in umbilical cord

\begin{tabular}{lllllll}
\hline \multirow{2}{*}{ IL4 (categories) } & \multicolumn{2}{c}{ Experimental } & \multicolumn{2}{c}{ Control } & \multicolumn{2}{c}{ Total } \\
& $\mathbf{n}$ & $\%$ & $\mathbf{N}$ & $\%$ & $\mathbf{N}$ & $\%$ \\
\hline Undetectable & 19 & 70,37 & 8 & 29,63 & 27 & 43,55 \\
Detectable & 23 & 65,71 & 12 & 34,29 & 35 & 56,45 \\
Total & 42 & 67,74 & 20 & 32,26 & 62 & 100,00 \\
\hline
\end{tabular}

Table 7 Subjects group percentage distribution according to presence of maternal allergic records and IL4 Levels

\begin{tabular}{lllllll}
\hline \multirow{2}{*}{ Maternal Allergic Records } & \multicolumn{2}{c}{ IL4 Undetectable } & \multicolumn{2}{c}{ IL4 Detectable } & \multicolumn{2}{c}{ Total } \\
& N & \% & n & \% & n & \% \\
\hline Yes & 17 & 45,95 & 20 & 54,05 & 37 & 59,68 \\
No & 10 & 40,00 & 15 & 60,00 & 25 & 40,32 \\
Total & 27 & 43,55 & 35 & 56,45 & 62 & 100,00 \\
\hline
\end{tabular}

Regarding IL4 determination, the relation to the maternal allergic pathologies record did not show a significant relevance in the 35 subjects with detectable IL4 values.

Citation: Aurora RAL, Mayerling ZGM, DelValle TJ, et al. Levels of interleukin 4 and immunoglobulin E in umbilical cord blood from children born to allergic mothers. MOJ Immunol. 20I 7;5(6): I I-I2. DOI: I0.15406/moji.20I7.05.00I75 
Table 8 Subject group percentage distribution according to ige levels in umbilical cord and il4 levels. As for the IL4 levels in umbilical cord blood, it can be considered that only I of the 2 subjects who had IgE values higher than $10 \mathrm{IU}$ levels in cord had detectable IL4 values

\begin{tabular}{|c|c|c|c|c|c|c|}
\hline \multirow{2}{*}{ IgE Cord (categories) } & \multicolumn{2}{|c|}{ IL4 Undetectable } & \multicolumn{2}{|c|}{ IL4 Detectable } & \multicolumn{2}{|c|}{ Total } \\
\hline & $\mathbf{N}$ & $\%$ & $\mathbf{N}$ & $\%$ & $\mathbf{N}$ & $\%$ \\
\hline Low values $(-10)$ & 26 & 43,33 & 34 & 56,67 & 60 & 96,77 \\
\hline High values (+ I0) & I & 50,00 & I & 50,00 & 2 & 3,23 \\
\hline Total & 27 & 43,55 & 35 & 56,45 & 62 & 100,00 \\
\hline
\end{tabular}

Table 9 Subject Group Percentage Distribution According To Ige Levels (3 Months) And Control Group (Children Born To Non-Allergic Mothers)

\begin{tabular}{|c|c|c|c|c|c|c|}
\hline \multirow{2}{*}{ IgE 3 months (categories) } & \multicolumn{2}{|c|}{ Experimental } & \multicolumn{2}{|c|}{ Control } & \multicolumn{2}{|c|}{ Total } \\
\hline & $\mathbf{N}$ & $\%$ & $\mathbf{N}$ & $\%$ & $\mathbf{N}$ & $\%$ \\
\hline Low values $(-10)$ & 6 & 100,00 & 0 & 0,00 & 6 & 37,50 \\
\hline High values (+ I0) & 6 & 60,00 & 4 & 40,00 & 10 & 62,50 \\
\hline Total & 12 & 75,00 & 4 & 25,00 & 16 & 100,00 \\
\hline
\end{tabular}

Only 6 of the 16 subjects of the experimental group who completed the study had levels above 10 IU.

Table 10 Subject group percentage distribution according to il4 levels ( 3 months) and control group (children born to non-allergic mothers)

\begin{tabular}{lllllll}
\hline \multirow{2}{*}{ months IL4 (categoríes) } & \multicolumn{2}{c}{ Experimental } & \multicolumn{2}{c}{ Control } & \multicolumn{2}{c}{ Total } \\
& $\mathbf{N}$ & $\%$ & $\mathbf{n}$ & \% & N & $\%$ \\
\hline Undetectable & 7 & 70,00 & 3 & 30,00 & 10 & 62,50 \\
Detectable & 5 & 83,33 & 1 & 16,67 & 6 & 37,50 \\
Total & 12 & 75,00 & 4 & 25,00 & 16 & 100,00 \\
\hline
\end{tabular}

Only 5 of the 16 subjects of the experimental group who completed the study had detectable IL4 levels.

Table I I Subject group percentage distribution according to infant feeding and total ige values at the third month of life

\begin{tabular}{|c|c|c|c|c|c|}
\hline & & & \multicolumn{2}{|c|}{ IGE 3 months (categories) } & \multirow{2}{*}{ total } \\
\hline & & & Low values $(-10)$ & High values (+ I0) & \\
\hline \multirow{6}{*}{3 Months Feeding (infant) } & & Counting & 2 & 4 & 6 \\
\hline & Exclusive breastfeeding & \%Within 3 Months Feeding (infant) & $33,3 \%$ & $66,7 \%$ & $100,0 \%$ \\
\hline & & \% Within 3 months IgE (categories) & $33,3 \%$ & $40,0 \%$ & $37,5 \%$ \\
\hline & & Counting & 4 & 6 & 10 \\
\hline & Mixed breastfeeding & \% Within 3 Months Feeding (infant) & $40,0 \%$ & $60,0 \%$ & $100,0 \%$ \\
\hline & & \%Within 3 Months IgE (categories) & $66,7 \%$ & $60,0 \%$ & $62,5 \%$ \\
\hline \multirow{3}{*}{\multicolumn{2}{|c|}{ Total }} & Count & 6 & 10 & 16 \\
\hline & & \%Within 3 Months Feeding (infant) & $37,5 \%$ & $62,5 \%$ & $100,0 \%$ \\
\hline & & \% Within 3 Months IgE (categories) & $100,0 \%$ & $100,0 \%$ & $100,0 \%$ \\
\hline
\end{tabular}

Only 6 of the 16 subjects who completed the study presented mixed breastfeeding feeding and IgE Total values higher than I0 IU.Variables: gender, childbirth, Family Records, IL4 and IgE Levels and Presence of Eczema.

Table 12 Subjects groups characterization during 3 consecutive months

\begin{tabular}{|c|c|c|c|c|c|c|c|c|c|c|}
\hline $\begin{array}{l}\text { Subject } \\
\text { group }\end{array}$ & Sex & $\begin{array}{l}\text { Labor } \\
\text { complications }\end{array}$ & $\begin{array}{l}\text { Maternal } \\
\text { Records }\end{array}$ & $\begin{array}{l}\text { Paternal } \\
\text { Records }\end{array}$ & IL4 & $\begin{array}{l}\text { IL4 (3 } \\
\text { Months) }\end{array}$ & $\begin{array}{l}\text { IgE } \\
\text { Cord }\end{array}$ & $\begin{array}{l}\text { IgE } \\
\text { Maternal }\end{array}$ & $\begin{array}{l}\text { IgE (3 } \\
\text { Month) }\end{array}$ & $\begin{array}{l}\text { Eczema } \\
\text { Presence }\end{array}$ \\
\hline \multirow{12}{*}{ Experimental } & \multirow{3}{*}{ Male } & Yes & Yes & No & Detectable & Undetectable & High & Low & Low & No \\
\hline & & No & Yes & Yes & Undetectable & Undetectable & High & High & High & No \\
\hline & & Yes & No & No & Detectable & Detectable & High & Low & High & Yes \\
\hline & \multirow{9}{*}{ Female } & No & No & Yes & Undetectable & Undetectable & High & Low & Low & Yes \\
\hline & & No & Yes & No & Detectable & Undetectable & High & Low & Low & Yes \\
\hline & & Yes & Yes & No & Detectable & Undetectable & High & Low & Low & Yes \\
\hline & & No & Yes & No & Detectable & Detectable & High & High & Low & Yes \\
\hline & & No & Yes & No & Detectable & Detectable & High & Low & Low & Yes \\
\hline & & No & Yes & No & Detectable & Detectable & High & Low & High & No \\
\hline & & No & Yes & No & Undetectable & Detectable & High & Low & High & No \\
\hline & & No & Yes & Yes & Undetectable & Detectable & High & Low & High & No \\
\hline & & No & No & No & Detectable & Detectable & High & Low & High & No \\
\hline \multirow{4}{*}{ Control } & \multirow{2}{*}{ Male } & No & No & No & Undetectable & Detectable & High & Low & High & No \\
\hline & & Yes & No & No & Detectable & Undetectable & High & Low & High & Yes \\
\hline & \multirow{2}{*}{ Female } & No & No & No & Detectable & Detectable & High & Low & High & Yes \\
\hline & & No & No & No & Detectable & Detectable & High & Low & High & No \\
\hline
\end{tabular}




\section{Discussion}

Immunoglobulin $\mathrm{E}$ is the least frequent antibody present in serum, with a normal concentration of 50-200 IU in non-allergic individuals. Serum Immunoglobulin E half-life is 3 days. However, much of Immunoglobulin $\mathrm{E}$ remains in the tissues, in contrast to serum Immunoglobulin $\mathrm{E}$; this is bound to the cell receptor having a half-life of a few weeks. The Immunoglobulin E expression is produced by a heavy chain change from an Immunoglobulin $\mathrm{M}$, by somatic recombination of the genes in the B cell germ line in a micro Th2 environment represented by Interleukin 4, 5, 9 and 13. In our country, there is a representative number of allergic diseases that increase day by day and that affect our pediatric population, that is why the present study is important in evaluating and determining the levels of Interleukin 4 and Immunoglobulin E in umbilical cord blood of children born to mothers with allergy family record and the $\operatorname{IgE}$ association with allergic disease onset in the first trimester of life. The determination of $\operatorname{IgE}$ and Interleukin 4 by the indirect Elisa method in blood remains the most specific and sensitive method for patients with an allergic records evaluation, which represents an allergic disease predictive value. In this regard, levels of Immunoglobulin E are low in umbilical cord blood; however, their detection demonstrates fetus's ability to produce this immunoglobulin, which does not cross the placenta. The immune response during fetal life and the neonatal period is predominantly Th-2 type. ${ }^{11}$ The placenta constitutes a selective barrier between the maternal and fetal circulation.

Recently, it has been revealed that maternal Ig E antibodies are present in macrophages of the human placenta villi regardless maternal allergic status. Regarding the allergic maternal record, it should be noted that in this study there was no significant statistical relevance with elevated IgE levels in subject's umbilical cord blood; atopy development occurs in $30 \%$. These results are similar to studies in Europe and the United States. ${ }^{10}$ Those studies assent the following: if family record is positive and Immunoglobulin $\mathrm{E}$ in the umbilical cord is normal the development of atopy occurs in 30\%. Only $10 \%$ of children with a family negative record and high Immunoglobulin E in umbilical cord will present atopy. Therefore, family record (the inherited genetic component) is the most reliable predictor; Immunoglobulin $\mathrm{E}$ in umbilical cord by itself is a parameter of very little sensitivity as a predictive factor. Even though, among allergic maternal records, allergic diseases such as bronchial asthma and allergic rhinitis were found in almost half of the subjects.

Among the genetic aspects that predominate as predictive of allergic diseases we have: atopy family record and chromosome markers 5, 16, 6 and $11 .{ }^{14}$ In this study 13 subjects in the experimental group had a paternal record of allergic diseases and only one of them had IgE, high levels in umbilical cord blood; by contrast $50 \%$ of infants had siblings with allergic records, which suggests a probability of developing allergic manifestations in early childhood. Inadequate maternal nutrition during gestation seems to be associated with unfavorable effects on allergies productions, together with a hereditary genetic record. The most common allergic maternal complications during pregnancy and childbirth were asthma and urticaria, asthma and rhinitis, respectively, which were correlated in this study with exacerbation and crisis observed in 2 patients in the experimental group. Regarding total Ig E, only 5 had total $\operatorname{IgE}$ levels above $150 \mathrm{IU}$, two of them in the control group with no allergic record, which shows other possible causes of elevation of IgE. IgE is not only involved in allergic inflammatory processes, but has also been associated with protection against parasitic infections. Studies in Gambia population revealed that there was an association between parasite-specific IgE, high levels and resistance to infection. As for mothers with IgE higher than $150 \mathrm{IU}$ values, only 2 of the subjects had elevated IgE in umbilical cord blood. This is corroborated in relation to the studies which mention that $50 \%$ of children born to allergic mothers had IgE high levels in umbilical cord blood. More than a half of the 42 subjects in the experimental group had detectable levels of Interleukin 4 as well as in the control group, suggesting that Interleukin 4 had a greater sensitivity and a positive predictive value for allergic disease development when compared to total $\operatorname{IgE}$ that has high specificity and low sensitivity.

Regarding the allergic epigenetic records and detectable Interleukin 4 values, 13 of the subjects had a paternal record; 5 of them had elevated Interleukin 4 levels detectable in umbilical cord blood. Likewise, when compared to the 2 subjects with umbilical cord IgE values above $10 \mathrm{IU}$, only 1 of them had detectable values of Interleukin 4, which did not reduce the predictive value of this cytokine. Therefore, it should be evaluated in later studies by measuring other cytokines of immune response. It should also be noted that 6 of the 16 subjects who completed 3 months of study in the experimental group had IgE levels higher than $10 \mathrm{IU}$ compared to less than a half who had detectable Interleukin 4. Five (5) were in the experimental group and one (1) was in the control group, which indicates the discordance in control group result since they did not have positive direct allergic records. This is the reason why it is considerably the follow up this patient to evaluate the development of allergic diseases in the early childhood. Similarly, out of the 16 subjects who completed the study, 10 presented IgE levels above $10 \mathrm{IU} ; 6$ received mixed breastfeeding compared to 4 who received exclusive breastfeeding. More than a half have high $\operatorname{IgE}$ values which corroborates that early milk formulas introduction promotes immune system alteration in subjects with positive epigenetic records. During the first months of life, a high percentage of atopic individuals may manifest allergic disease; in general, atopic dermatitis is the primary manifestation. Children with atopic dermatitis who started breastfeeding- in addition to a family allergy record had a greater risk of developing asthma and (or) other allergic subsequent manifestations. In this study it was evident that 6 of the 16 subjects who completed the study in the experimental group presented atopic dermatitis; 2 subjects in the control group also presented atopic dermatitis which represents $50 \%$ of the subjects. This is a significant statistical value which leads to promote studies where allergic manifestations appearance can be evaluated for a longer time.

\section{Conclusion}

According to the study, we can settle:

a. There is statistical significance regarding atopy family record in these subjects as a predictive value of allergy development during the first trimester of life. It is evidence that the isolated parameter of Immunoglobulin E in umbilical cord blood is less sensitive as the predictive value of allergic disease in this age group.

b. Subjects with a record of allergic disease in their siblings also had a predisposition to develop allergies in the first trimester of life, which is evident in half of the subjects who completed the study. In these cases, the record had statistical significance.

c. Close to $30 \%$ of the subjects with a maternal allergic record in the experimental group had a paternal allergic record which is significant; this condition is considered favorable for allergic pathology development.

d. In the experimental group, whose mothers had elevated $\operatorname{IgE}$ levels, there was no relationship neither with elevated IgE levels in umbilical cord blood of newborn children or with subsequent 
development of allergic disease during the first trimester of life, which could be explained by the high specificity but low sensitivity of IgE.

e. Subjects who had detectable levels of Interleukin 4 in umbilical cord blood persisted with detectable values at 3 months of life, suggesting that these values are much more sensitive as predictors of future allergy development.

f. High levels of IgE in umbilical cord blood were found in a subject with no maternal allergy record, which could be explained by the fact that the production of this molecule belongs to the newborn and does not depend on circulating $\operatorname{IgE}$ maternal values.

g. Subjects with no allergic disease family records who had high IgE levels developed dermatological clinical manifestations, such as eczema, during the first three months of life.

h. Noteworthy, in recent studies, the maternal record of asthma was correlated with IgE and interleukin 4 high levels in the umbilical cord, increasing the risk of developing food allergies in the first year of life.

i. There was no statistically significant correlation between elevated IgE level, allergic disease record and subsequent development of allergic pathologies in children during the first trimester of life.

j. Interleukin 4 values have higher predictive value taken in umbilical cord blood, compared to a control after three months of life taken in peripheral blood.

k. High levels of total IgE in umbilical cord blood, without family atopy record, have no statistical significance as predictive allergic disease value during the first trimester. However, a high IgE value associated with Interleukin 4 detectable levels, additionally related to an environmental exposure to allergens, predicts the development of allergic pathology.

1. In this study, Interleukin 4 detectable values related to atopy family record, predict allergic pathology development during the first trimester of life.

$\mathrm{m}$. IgE is a newborn valuable parameter which does not depend on IgE maternal production during pregnancy.

n. It is important to remember that there are other laboratory parameters that can be evaluated to determine a patient allergic status, such as eosinophils, Interleukin 10, Interleukin 2, Interferon gamma and lymphocyte subpopulation.

o. The present study provides the basis for future research in this area, contributing to a deeper understanding of this theoretical theme.

\section{Acknowledgments}

The authors received no financial assistance for the research.

\section{Conflicts of interest}

The author has no conflicts of interest.

\section{References}

1. GINA. Global strategy for asthma management and prevention. WHO/ NHLBI Workshop Report. National Institute of Health, USA. 2004.

2. World Allergy Organization. Guidelines for prevention of allergic asthma. Allergy Clinical Immunology Int J. 2004;16(5):176-186.

3. Scirica C, Gold DR, Ryan L, et al. Predictors of IgE in umbilical cord blood in children at asthma and atopy risk. J Allergy Clin Immunol. 2007;119(1):81-88.

4. Bapat M, Shah S. Cord serum screening test and the newborn allergic status. Indian Pediatr. 2009;46(4):295-299.

5. Sybilski AJ, Doboszynska A, Samolinski B. Prediction of atopy in the first year of life using cord blood IgE levels and family history. Eur $J$ Med Res. 2009;14 (Suppl 4):227-232.

6. Sandberg M, Frykman A, Ernerudh J, et al. Cord blood cytokines and chemokines and development of allergic disease. Pediatr Allergy Immunol. 2009;20(6):519-527.

7. Sybilski AJ, Doboszynska A, Samolinski B. Total and antigen-specific IGE levels in umbilical cord blood. Eur J Med Res. 2009;14(Suppl 4):233-236

8. Cárdenas P, Fernández L, Col Y. Levels of interleukins in cord blood:relationship with allergic disease family record. Spanish Society of Clinical Immunology and Pediatric Allergology. 2008;4(6).

9. Rindsjo, E, Joerink M, Papadogiannakis N, et al. IgE in the human placenta:why there? Allergy. 2010;65(5):554-560.

10. Peters JL, Suglia SF, Platts-Mills TA, et al. Relationships among prenatal aeroallergen exposure and maternal and cord blood IgE:project ACCESS. J Allergy Clin Immunol. 2009;123(5):1041-1046.

11. Vilchis P, Becerril M. IgE concentrations in umbilical cord blood and its association with atopic risk factors. Education and Clinical Research. 2016;1(3):172-181.

12. Kaplan A, Bousquet J. Prevention of allergy asthma. Allergy clinical Immunology. 2009;12(6):288-299.

13. http://www.24hsitges.com/attachments/068_sitges\%202003-11.pdf

14. Kim JH, Kim KH, Woo HY, Shim JY. Maternal cytokine production during pregnancy and the development of childhood wheezing and allergic disease in offspring three years of age. $J$ Asthma. 2008;45(10):948-952.

15. Paz Alvarez S. Total IgE Determination In Cord Blood Like Predictive Factor And Risk On The Development Of Allergy Illness In Newborns Of Parents Had History Of Atopy. Medical Journal of Postgraduate Medicine UNAH. 2007;10(1).

16. Cárdenas Guerrero P, Fernández Lorenzo JM, Martínez-Cañavate Burgos A, et al. Interlukin levels in umbilical cord blood:relationship with a family history of allergic disease. Allergol Immunopathol. 2005;33(3):131-137.

17. Lawrence R. Long-term effects on allergy prophylaxis. Education in breastfeeding. (6th edn), Elsevier, USA. 2007. pp. 769-776.

18. Arias, Fidias. The Research Project. Introduction to the Scientific Methodology (5th edn), Editorial Episteme. Caracas, Venezuela. 2006. p. 21-36.

\section{Funding}

None. 